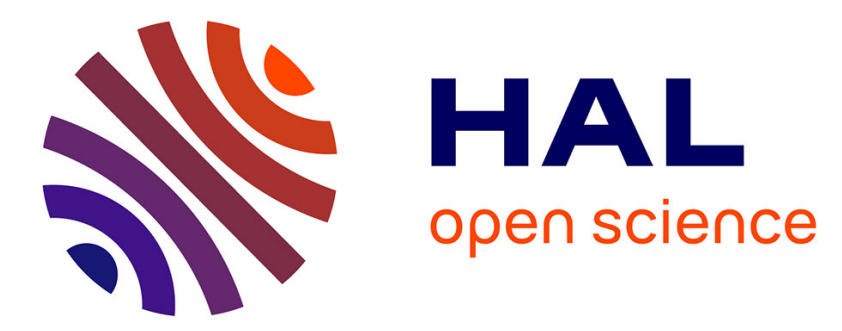

\title{
Backward Parabolicity, Cross-Diffusion and Turing Instability
}

Ayman Moussa, Benoît Perthame, Delphine Salort

\section{To cite this version:}

Ayman Moussa, Benoît Perthame, Delphine Salort. Backward Parabolicity, Cross-Diffusion and Turing Instability. Journal of Nonlinear Science, 2018, 29 (1), pp.139-162. 10.1007/s00332-018-9480-z . hal-01626324

\section{HAL Id: hal-01626324 \\ https://hal.science/hal-01626324}

Submitted on 30 Oct 2017

HAL is a multi-disciplinary open access archive for the deposit and dissemination of scientific research documents, whether they are published or not. The documents may come from teaching and research institutions in France or abroad, or from public or private research centers.
L'archive ouverte pluridisciplinaire HAL, est destinée au dépôt et à la diffusion de documents scientifiques de niveau recherche, publiés ou non, émanant des établissements d'enseignement et de recherche français ou étrangers, des laboratoires publics ou privés. 


\title{
Cross-diffusions and Turing instabilities
}

\author{
Ayman Moussa*† \\ Benoît Perthame ${ }^{* \dagger} \S$ \\ Delphine Salort ${ }^{\dagger \S}$
}

October 29, 2017

\begin{abstract}
We show that the ill-posedness observed for cross-diffusion systems, that is due to backward parabolicity, can be interpreted as a limiting Turing instability of a corresponding semi-linear parabolic system. Our analysis is based on the, now well established, derivation of cross-diffusions from reaction-diffusion systems for fast reaction rates.

We illustrate our observation with two generic examples for $2 \times 2$ and $4 \times 4$ reaction-diffusion systems. For these examples, we prove that backward parabolicity in cross-diffusion systems is equivalent to Turing instability for fast reaction rates. In one dimension, the Turing patterns are periodic solutions which frequency increases with the reaction rate. Furthermore, in some specific cases, the structure of the equations at hand involves classical entropy/Lyapunov functions which lead to a priori estimates allowing to rigorously pass to the fast reaction limit in the absence of Turing instabilities.
\end{abstract}

2010 Mathematics Subject Classification. 35B36; 35B10; 35K57; 35Q92

Keywords and phrases. Reaction-diffusion systems; Cross-diffusion systems; Turing instability; Backward parabolic equations; Mathematical biology; Entropy; Periodic solutions;

\section{Introduction and model equations}

The derivation of cross-diffusion dynamic from fast reactions in parabolic systems, after it was observed in [10, 13], has known a growing interest during the past few decade [5, 4, 7, 1, 20] and the subject is well established.

Here, we consider specific form of cross-diffusion systems as in

$$
\partial_{t} w_{i}-\Delta\left[A_{i}(w)\right]=0, \quad i=1, \ldots, I,
$$

\footnotetext{
*Sorbonne Universités, UPMC Univ Paris 06, Laboratoire Jacques-Louis Lions UMR CNRS 7598, F75005 Paris, France

${ }^{\dagger}$ The research leading to this paper was (partially) funded by the french "ANR blanche" project Kibord: ANR-13BS01-0004

${ }^{\ddagger}$ Inria de Paris, EPC Mamba, F75005 Paris, France. BP has received funding from the European Research Council (ERC) under the European Union Horizon 2020 research and innovation programme (grant agreement No 740623)

$\S$ This author wishes to thank Antoine Triller, Institut de Biologie de l'ENS, for several discussions which motivated this research line.

`Sorbone Universités, UPMC, UMR 7238, Laboratoire de Biologie Computationnelle et Quantitative. Email: delphine.salort@upmc.fr
} 
where the nonlinearity $A$ depends on the solution vector $w=\left(w_{1}, \ldots, w_{I}\right)$ and satisfies $\left.A_{i}(w)\right|_{w_{i}=0}=0$. We aim at proving the equivalence between instability by backward parabolicity for these crossdiffusion equations and Turing instabilities for fast reaction-diffusion systems. Let us point out that this type of instability is very different from that mentioned already in [10, 13] which is the continuation of Turing instability through their asymptotic process when some reaction terms remain in the crossdiffusion system, as it is the case in the famous SKT model [25, 6].

Our motivation comes from the fast dynamics of attachment and detachment of synaptic receptors to scaffold proteins which has been observed and modeled in [24, 9], leading to large dynamical aggregates [22]. This attachment and detachment dynamics has been analyzed, with a different scaling than that we propose here, in [11].

For our purpose, we first consider the simple case of two coupled equations with mass conservation leading formally to a nonlinear diffusion equation when the reaction rate goes to infinity. Then, we extend our analysis to the setting of four coupled reaction-diffusion equations with mass conservation, leading formally, when the reaction rate goes to infinity, to two coupled cross-diffusion equations.

We now describe more precisely the two settings of our study, the main general properties of the involved equations and the limit equation obtained heuristically when the fast of the reaction term goes to $+\infty$.

\subsection{Case of 2 coupled reaction-diffusion equations}

We begin with a very simple example. We consider a smooth bounded domain $\Omega \subset \mathbb{R}^{d}$ and nongative solutions of the the singular perturbation problem with Neumann boundary conditions

$$
\left\{\begin{array}{l}
\partial_{t} u_{\varepsilon}-d_{1} \Delta u_{\varepsilon}=\varepsilon^{-1}\left(v_{\varepsilon}-F\left(u_{\varepsilon}\right)\right), \quad x \in \Omega, t \geq 0 \\
\partial_{t} v_{\varepsilon}-d_{2} \Delta v_{\varepsilon}=-\varepsilon^{-1}\left(v_{\varepsilon}-F\left(u_{\varepsilon}\right)\right), \\
\frac{\partial u_{\varepsilon}}{\partial \nu}=\frac{\partial v_{\varepsilon}}{\partial \nu}=0, \quad \text { on } \partial \Omega,
\end{array}\right.
$$

where the diffusion coefficients satisfy $d_{1}>0, d_{2}>0$ and

$$
F \in \mathscr{C}^{2}\left(\mathbb{R}_{+} ; \mathbb{R}_{+}\right) \text {satisfies } F^{\prime}>-1, \quad F(0)=0, \quad F(u)>0 \text { for } u>0
$$

With these assumptions, solutions remain non-negative, and it is also convenient to impose an upper bound $u \leq u_{M}, v \leq v_{M}=F\left(u_{M}\right)$ for some $u_{M}$ such that

$$
v_{M} \geq F(u) \quad \forall u, 0 \leq u \leq u_{M}
$$

In particular the set of equibria is parametrized by the equation $v=F(u)$. The small parameter $\varepsilon>0$ measures the time scale of reaction compared to diffusion.

This particular setting is also used to model mechanisms of cell polarisation [12], [19], and have generated many mathematical contributions [23, 17, 15, 16]. Indeed, the authors, see [17] and references therein, exhibit specific entropy functionals that we recall below and allow for a full asymptotic theory.

As specified, summing the two equations, we obtain the first basic property of this system, that is the conservation law,

$$
\partial_{t} w_{\varepsilon}-\Delta\left[d_{1} u_{\varepsilon}+d_{2} v_{\varepsilon}\right]=0, \quad w_{\varepsilon}=u_{\varepsilon}+v_{\varepsilon}
$$


which implies that

$$
\int_{\Omega}\left(u_{\varepsilon}(x, t)+v_{\varepsilon}(x, t)\right) d x=: M \quad \text { is independent of } t .
$$

Heuristically, when $\varepsilon \rightarrow 0$, we expect that $\left(u_{\varepsilon}, v_{\varepsilon}\right) \rightarrow(u, v)$, and $v=F(u)$. Therefore, from (3), we get

$$
\partial_{t} w-\Delta\left(d_{1} u+d_{2} F(u)\right)=0, \quad w=u+F(u) .
$$

The condition (2) implies that one can invert the mapping $u \mapsto w=u+F(u)$, and thus write

$$
d_{1} u+d_{2} F(u)=A(w), \quad A \in \mathscr{C}^{1}\left(\mathbb{R}^{+} \times \mathbb{R}^{+}\right),
$$

which formally generates the equation

$$
\left\{\begin{array}{l}
\partial_{t} w-\Delta A(w)=0, \quad x \in \Omega, t \geq 0 \\
\frac{\partial w}{\partial \nu}=0, \quad \text { on } \partial \Omega
\end{array}\right.
$$

Notice that we have

$$
A^{\prime}(w)=\frac{d_{1}+d_{2} F^{\prime}(u)}{1+F^{\prime}(u)}, \quad w=u+F(u) .
$$

Using the assumption (2), when $d_{2} / d_{1} \leq 1$, this equation is parabolic, of porous medium type, and there is an entropy/Lyapunov functional, i.e., a functional of $u_{\varepsilon}, v_{\varepsilon}$ which is decreasing with time if $F^{\prime} \geq-\frac{d_{2}}{d_{1}}$ (see Proposition 2.8.

Our goal is to give conditions on $F$, around a given constant steady state $(\bar{u}, \bar{v})$, that means $\bar{v})=F(\bar{u})$, for which the following holds. For $d_{2} / d_{1}$ large enough and $\varepsilon$ small enough, the state $(\bar{u}, \bar{v})$ is Turing unstable for (1), and this turns out to be equivalent to the backward parabolicity of the limiting equation, which means $A^{\prime}(\bar{w})<0$.

We recall that the forward-backward parabolic Equation (5) is ill-posed under the condition $A^{\prime}(\bar{w})<$ 0 , however this problem has attracted a lot of interest and a theory of Young-measure solutions could certainly be derived along the lines of [18, 2, 21, 8, 26, 14].

\subsection{Case of 4 coupled reaction-diffusion equations}

Next, we extend the method to the case of four coupled equations, leading, in the fast reaction rate limit, to a $2 \times 2$ cross-diffusion equations. We explore again the correspondance between Turing instability and backward parabolicity in the cross-diffusion system equation.

We consider the following system, built with symmetry between two sets of variables,

$$
\left\{\begin{array}{l}
\partial_{t} u_{\varepsilon}^{1}-d_{1} \Delta u_{\varepsilon}^{1}=\frac{1}{\varepsilon}\left[R\left(v_{\varepsilon}, w_{\varepsilon}\right)+\frac{u_{\varepsilon}^{2}}{2}-\frac{u_{\varepsilon}^{1}}{2}\right], \\
\partial_{t} u_{\varepsilon}^{2}-d_{2} \Delta u_{\varepsilon}^{2}=-\frac{1}{\varepsilon}\left[R\left(v_{\varepsilon}, w_{\varepsilon}\right)+\frac{u_{\varepsilon}^{2}}{2}-\frac{u_{\varepsilon}^{1}}{2}\right], \\
\partial_{t} u_{\varepsilon}^{3}-d_{3} \Delta u_{\varepsilon}^{3}=\frac{1}{\varepsilon}\left[S\left(v_{\varepsilon}, w_{\varepsilon}\right)+\frac{u_{\varepsilon}^{4}}{2}-\frac{u_{\varepsilon}^{3}}{2}\right] \\
\partial_{t} u_{\varepsilon}^{4}-d_{4} \Delta u_{\varepsilon}^{4}=-\frac{1}{\varepsilon}\left[S\left(v_{\varepsilon}, w_{\varepsilon}\right)+\frac{u_{\varepsilon}^{4}}{2}-\frac{u_{\varepsilon}^{3}}{2}\right],
\end{array}\right.
$$


with

$$
v_{\varepsilon}=\frac{u_{\varepsilon}^{1}+u_{\varepsilon}^{2}}{2}, \quad w_{\varepsilon}=\frac{u_{\varepsilon}^{3}+u_{\varepsilon}^{4}}{2} .
$$

This system can be seen as the extension of the system (1) with the modification that the nonlinearity $F(u+v)-v$ replaces $F(u)-v$. The corresponding equilibrium, which cancel the right hand side, turns out to be defined by

$$
\begin{cases}u^{1}=v+R(v, w), & u^{2}=v-R(v, w), \\ u^{3}=w+S(v, w), & u^{4}=w-S(v, w) .\end{cases}
$$

We immediately see that, formally, the corresponding cross-diffusion system is given by

$$
\left\{\begin{array}{l}
\partial_{t} v-\Delta A(v, w)=0, \\
\partial_{t} w-\Delta B(v, w)=0
\end{array}\right.
$$

with

$$
A(v, w)=\frac{d_{1}+d_{2}}{2} v+\frac{d_{1}-d_{2}}{2} R(v, w), \quad B(v, w)=\frac{d_{3}+d_{4}}{2} w+\frac{d_{3}-d_{4}}{2} S(v, w) .
$$

At this stage, it is useful to introduce some assumptions. In order to preserve positivity, we assume (consider first that $u_{\varepsilon}^{1}$ vanishes and then $u_{\varepsilon}^{2}$, and argue in the same way for $u_{\varepsilon}^{3}$ and $u_{\varepsilon}^{4}$ ),

$$
\begin{array}{cc}
-v \leq R(v, w) \leq v, & -w \leq R(v, w) \leq w, \\
-v \leq S(v, w) \leq v, & -w \leq S(v, w) \leq w .
\end{array}
$$

Additionally, it is convenient to control solution with the maximum principle. That is the case when there exists $u_{M}>0$ such that

$$
\begin{array}{ll}
\frac{u-u_{M}}{2} \leq R\left(\frac{u+u_{M}}{2}, w\right) \leq \frac{u_{M}-u}{2}, \quad \forall w \geq 0, \forall 0 \leq u \leq u_{M}, \\
\frac{u-u_{M}}{2} \leq S\left(v, \frac{u+u_{M}}{2}\right) \leq \frac{u_{M}-u}{2}, \quad \forall v \geq 0, \forall 0 \leq u \leq u_{M} .
\end{array}
$$

Finally, we want that the self-diffusion is always positive and that instabilities stem from the crossterms

$$
A_{v}>0 \Leftrightarrow\left(d_{1}+d_{2}\right)+\left(d_{1}-d_{2}\right) R_{v}>0, \quad B_{w}>0 \Leftrightarrow\left(d_{3}+d_{4}\right)+\left(d_{3}-d_{4}\right) S_{w}>0 .
$$

Again, for a given constant steady state, our goal is to prove equivalence between Turing instability for (6) and backward parabolicity for the cross-diffusion system (7). However, unlike the case system (1), for the $4 \times 4$ system, there does not exist entropy functionals in general, except in specific settings that we discuss in section 3.2 .

\subsection{Organisation of the paper}

In section 2, we consider the system (1). We first prove that, being given a constant steady state, there is equivalence between Turing instabilities when $\varepsilon$ is small enough and backward parabolicity in (5). Then, we study the different possible non-constant steady states with respect to $\varepsilon$. We exhibit a wide family of periodic steady states and study their isochronous character that may be induced by 
$F$. Finally, we exhibit some classical entropy inequalities which give results in accordance with the criteria obtained on the function $F$ for the existence of Turing instabilities.

In section 3, we extend our analysis to the system (6). We show that, again, there is equivalence between asymptotic Turing instabilities for $\varepsilon$ small and he backward parabolicity for the cross-diffusion terms in the equation (8). The last part of this section is devoted to the existence of an entropy functional in specific settings.

\section{Turing instability and analysis of Equation (1)}

We begin with several aspects of the fast reaction in the system (1) under assumpation (2). We plan to, on the one hand, understand the set of non constant steady states in dimension 1 , and on the other hand, interpret our results with respect to some well-known entropy/Lyapunov structure on those equations [17].

\subsection{Turing instability of system (1) and backward parabolic equation}

To tackle the question of the equivalence between Turing instability and backward parabolicity for Equation (1), we consider a constant steady state $(\bar{u}, \bar{v}), \bar{v}=F(\bar{u})$ and we recall some basic observations and definitions.

Firstly, we consider the dynamical system

$$
\frac{d}{d t}\left(\begin{array}{l}
u \\
v
\end{array}\right)=\left(\begin{array}{l}
v-F(u) \\
F(u)-v
\end{array}\right)
$$

Because the quantity $M:=u(t)+v(t)$ is constant, the solution is reduced to the simple equation $\frac{d u}{d t}=M-u-F(u)$. Therefore, the state $(\bar{u}, \bar{v})$ is attractive for initial data satisfying $M=F(\bar{u})+\bar{u}$ thanks to the assumption (2).

Around such a steady state $\bar{U}$, the linearized system reads

$$
\left\{\begin{array}{l}
\partial_{t} \delta u_{\varepsilon}-d_{1} \Delta \delta u_{\varepsilon}=\varepsilon^{-1}\left[\delta v_{\varepsilon}-F^{\prime}(\bar{u}) \delta u_{\varepsilon}\right], \\
\partial_{t} \delta v_{\varepsilon}-d_{2} \Delta \delta v_{\varepsilon}=\varepsilon^{-1}\left[F^{\prime}(\bar{u}) \delta u_{\varepsilon}-\delta v_{\varepsilon}\right] .
\end{array}\right.
$$

Its stability can be analyzed by decomposition on the spectral basis $\left(w_{i}\right)_{i \in \mathbb{N}}$ associated with the Laplacian

$$
-\Delta w_{i}=\lambda_{i} w_{i} \quad \text { in } \Omega \quad \frac{\partial w_{i}}{\partial \nu}=0 \quad \text { on } \partial \Omega .
$$

Considering the projections $\left(\alpha_{i}, \beta_{i}\right)=\int_{\Omega}\left(\delta u_{\varepsilon}, \delta v_{\varepsilon}\right) w_{i} d x$, the system 15 is written as linear independent equations

$$
\left\{\begin{aligned}
\frac{d \alpha_{i}}{d t}+d_{1} \lambda_{i} \alpha_{i} & =\varepsilon^{-1}\left[\beta_{i}-F^{\prime}(\bar{u}) \alpha_{i}\right], \\
\frac{d \beta_{i}}{d t}+d_{2} \lambda_{i} \beta_{i} & =\varepsilon^{-1}\left[F^{\prime}(\bar{u}) \alpha_{i}-\beta_{i}\right] .
\end{aligned}\right.
$$

Definition 2.1 We say that the steady state $(\bar{u}, \bar{v})$ is asymptotically Turing unstable if, for $\varepsilon$ small enough, it is Turing instable for the system (1), i.e., one of the components $i \in \mathbb{N}$ of the system (16) has a uniformly negative real part for $\varepsilon$ small enough.

The following result holds 
Proposition 2.2 (Equivalence in the scalar case.) We assume (2) and consider a steady state $(\bar{u}, \bar{v})$. It is asymptotically Turing unstable for (1), if and only if the equation (5) is backward parabolic that is if $A^{\prime}(\bar{u}+F(\bar{u}))<0$ or also

$$
d_{1}+d_{2} F^{\prime}(\bar{u})<0 \text {. }
$$

Remark 2.3 It will be clear from our proof below that this result still holds replacing $v-F(u)$ by a function $\phi(u, v)$ with adequate assumptions. Namely for $u \geq 0, v \geq 0$ one should impose $\phi(0, v) \geq 0$, $\phi(u, 0) \leq 0$, for some $u_{M}>0 \phi\left(u_{M}, v\right) \leq 0$ and $\phi\left(u, u_{M}\right) \geq 0, \partial_{v} \phi(u, v)=0$ and $\phi(u, F(u))=0$.

Proof. To characterize asymptotic Turing instability we look for a value $\lambda$ with $\operatorname{Re} \lambda>0$ such that $\left(\alpha_{i}(t), \beta_{i}(t)\right)=e^{\lambda t}\left(\bar{\alpha}_{i}, \bar{\beta}_{i}\right)$. This is written

$$
\left\{\begin{array}{l}
\lambda \bar{\alpha}_{i}+d_{1} \lambda_{i} \bar{\alpha}_{i}=\varepsilon^{-1}\left[\bar{\beta}_{i}-F^{\prime}(\bar{u}) \bar{\alpha}_{i}\right] \\
\lambda \bar{\beta}_{i}+d_{2} \lambda_{i} \bar{\beta}_{i}=\varepsilon^{-1}\left[F^{\prime}(\bar{u}) \bar{\alpha}_{i}-\bar{\beta}_{i}\right]
\end{array}\right.
$$

This system can be reduced to $\lambda\left(\bar{\alpha}_{i}+\bar{\beta}_{i}\right)+\lambda_{i}\left(d_{1} \bar{\alpha}_{i}+d_{2} \bar{\beta}_{i}\right)=0$ and

$$
\lambda \bar{\alpha}_{i}+d_{1} \lambda_{i} \bar{\alpha}_{i}=\varepsilon^{-1}\left[-\bar{\alpha}_{i} \frac{\lambda+\lambda_{i} d_{1}}{\lambda+\lambda_{i} d_{2}}-F^{\prime}(\bar{u}) \bar{\alpha}_{i}\right] .
$$

A non-zero solution exists if and only if we can find a root to the polynomial

$$
\mathbb{P}(\lambda):=\varepsilon \lambda^{2}+\lambda\left(1+F^{\prime}(\bar{u})+\varepsilon \lambda_{i}\left(d_{1}+d_{2}\right)\right)+\varepsilon \lambda_{i}^{2} d_{1} d_{2}+\lambda_{i}\left(d_{1}+d_{2} F^{\prime}(\bar{u})\right) .
$$

If $d_{1}+d_{2} F^{\prime}(\bar{u}) \geq 0$ this convex polynomial with positive value at $\lambda=0$ and positive derivative at 0 cannot have a positive root and Turing instability is not possible.

If $d_{1}+d_{2} F^{\prime}(\bar{u})<0$, choose any eigenvalue $\lambda_{i}>0$ and $\varepsilon$ small enough such that

$$
2 \varepsilon \lambda_{i} d_{1} d_{2}<\left|d_{1}+d_{2} F^{\prime}(\bar{u})\right| .
$$

Then, it is immediate to check that there is a positive root with the form $\lambda=\lambda_{i} \Lambda\left(\varepsilon \lambda_{i}\right)$ which depends smoothly on $\varepsilon \lambda_{i}$ with $\Lambda(0)=-A^{\prime}(\bar{u}+F(\bar{u}))>0$ and thus, asymptotic turing instability holds.

\subsection{Study of stationary states}

We consider here the case of dimension 1 with $\Omega=(0, L)$ that is:

$$
\left\{\begin{array}{l}
\partial_{t} u_{\varepsilon}-d_{1} \Delta u_{\varepsilon}=\varepsilon^{-1}\left(v_{\varepsilon}-F\left(u_{\varepsilon}\right)\right) \\
\partial_{t} v_{\varepsilon}-d_{2} \Delta v_{\varepsilon}=-\varepsilon^{-1}\left(v_{\varepsilon}-F\left(u_{\varepsilon}\right)\right) .
\end{array}\right.
$$

We assume that $F \in C^{2}$ and, for some $u_{M}>0$,

$$
\begin{cases}F^{\prime}(\cdot)>-1, & F^{\prime}(0)>0, \quad F^{\prime}(u)>0 \quad \text { for } u \geq u_{M}, \\ F(u)<F\left(u_{M}\right) & \forall u<u_{M},\end{cases}
$$

Recalling the characterisation (17) of asymptotic Turing instability that we established previously, we consider the assumption

$$
d_{1}+d_{2} F^{\prime}(u) \geq 0, \quad \forall u \geq 0 .
$$

The following description for the stationary states of the system $(18)$ holds. 
Theorem 2.4 Assume $L>0$ given. We have two possible outcomes

- If (20) is satisfied, then any stationary state of (18) is constant.

- If (20) is not satisfied, let $\bar{u}$ such that $\frac{d_{1}}{d_{2}}+F^{\prime}(\bar{u})<0$ and $s_{\varepsilon}:=\sqrt{\varepsilon d_{1}}$. Then, there exists $T(\bar{u})$ such that if $L \in T(\bar{u}) s_{\varepsilon} \mathbb{N}$, there exists a non constant $T(\bar{u}) s_{\varepsilon}$ periodic steady state of (18) with $u(0)=\bar{u}$.

Remark 2.5 Let us mention, that in the above Theorem, we only prove existence of periodic solution for discret parameters $\varepsilon$. This is because the function $\frac{d_{1}}{d_{2}} u+F(u)$ may be isochronous [3]. A typical example is the case where $F$ is locally linear. Indeed, in this case, the solution u of (18), for initial data close enough to $\bar{u}$, are all proportional to

$$
\cos \left(s_{e}^{-1} \frac{2 \pi}{T(\bar{u})} x\right) .
$$

In Theorem 2.6 we give a simple criteria on the function $F$ in order to discard the isochronous setting.

Proof of Theorem 2.4. We first note that if $(u, v)$ are stationary states of Equation (18) then

$$
d_{1} u^{\prime \prime}+d_{2} v^{\prime \prime}=0
$$

and so, using the Neumann boundary condition, we also have

$$
d_{1} u^{\prime}+d_{2} v^{\prime}=0 .
$$

In particular, there exists $\lambda \in \mathbb{R}$ such that

$$
v=-\frac{d_{1}}{d_{2}} u+\lambda
$$

Plugging this in the equation satisfied by $u$ we get

$$
\begin{aligned}
u^{\prime \prime} & =\left(d_{1} \varepsilon\right)^{-1}\left(\frac{d_{1}}{d_{2}} u-\lambda+F(u)\right), \\
u^{\prime}(0) & =u^{\prime}(L)=0 .
\end{aligned}
$$

If 20 holds, then consider $w=u^{\prime}$ and differentiate (22) to get

$$
w^{\prime \prime}=\left(d_{1} \varepsilon\right)^{-1} w\left(\frac{d_{1}}{d_{2}} u+F^{\prime}(u)\right) .
$$

Integrating the previous equality against $w$ and using $w(0)=w(L)=0$ and using (20) leads to

$$
-\int_{0}^{L}\left|w^{\prime}(x)\right|^{2} d x \leq 0,
$$

so that $w=0$ and $u$ is constant (and so is $v$ ).

Now assume on the contrary that (20) does not hold. For any $\lambda \in \mathbb{R}$, if $u$ solves $(22)$ and $v$ is defined by (21), then $(u, v)$ is a stationary solution of (18). In particular, if $z$ solves

$$
\begin{aligned}
z^{\prime \prime} & =\frac{d_{1}}{d_{2}} z-\lambda+F(z), \\
z^{\prime}(0) & =z^{\prime}\left(L / s_{\varepsilon}\right)=0,
\end{aligned}
$$


then if $u(x):=z\left(x / s_{\varepsilon}\right)$ and $v$ is defined by (21), then $(u, v)$ is a stationary solution of (18). If $z$ is non constant and $T$-periodic, then $u$ and $v$ are non constant and $s_{\varepsilon} T$-periodic. We thus only need to prove the existence of $z$, a non constant periodic solution of (24)-(25). Since we are refuting (20), we have the existence $\bar{u}>0$ such that

$$
\frac{d_{1}}{d_{2}}+F^{\prime}(\bar{u})<0
$$

Define

$$
\lambda:=\frac{d_{1}}{d_{2}} \bar{u}+F(\bar{u})
$$

this means that $Y:=\left(z, z^{\prime}\right)$ solves the Hamiltonian system defined by the vector field $\Theta\left(y_{1}, y_{2}\right)=$ $\left(y_{2}, \mu\left(y_{1}\right)\right)$, where

$$
\mu\left(y_{1}\right):=\frac{d_{1}}{d_{2}} y_{1}-\lambda+F\left(y_{1}\right)
$$

We infer from Propositions A.1 of the Appendix the existence of (non constant) periodic solutions for our system. In order to conclude, we need to get a period $T$ such that $L \in s_{\varepsilon} T \mathbb{N}$. This ends the proof of Theorem 2.4.

\subsection{Continuum set of parameters with periodic solutions}

We now prove that there exists a continuum of periodic solutions with fixed total mass, assuming the following stronger assumption on $F$ : there exists a value $U$ in the unstable range, satisfying the conditions

$$
-1<F^{\prime}(U)<-\frac{d_{1}}{d_{2}}, \quad F^{\prime \prime}(U) \neq 0, \quad U+F(U)=M^{0} / L .
$$

More precisely, the following theorem holds.

Theorem 2.6 Assume that $F$ is smooth and that (26) holds. For all $n \in N^{*}$, there are non-constant periodic solutions with $n$ (minimal) periods and with mass $M^{0}$, defined when $\varepsilon$ belongs to a small interval $\left[\varepsilon_{n}, \varepsilon_{n}^{+}\right]$, where $\varepsilon_{n}=\frac{1}{d_{1}}\left(\frac{L}{n} \frac{\omega(U)}{2 \pi}\right)^{2}, \varepsilon_{n}<\varepsilon_{n}^{+}$and $\omega(U)=\sqrt{-\left(\frac{d_{1}}{d_{2}}+F^{\prime}(U)\right)}$.

Proof of Theorem 2.6. The idea of the proof of Theorem 2.6 is to consider a small perturbation of an unstable constant steady state $\bar{u}$ and to make some Taylors expansions in order to construct, modulo small order terms, explicitly the solution of (24)-25). This allow us to compute the Taylor expansion of the period and then to conclude the proof of our Theorem.

Let us first introduce the following notations

$$
y=x / \sqrt{\varepsilon d_{1}}, \quad w(y)=u(x), \quad V(u)=\frac{d_{1}}{d_{2}} \frac{u^{2}}{2}+G(u), \quad G(u)=\int_{0}^{u} F(s) d s .
$$

Then, we consider two parameters $(\bar{u}, \delta)$ with $\delta>0$ small and $\bar{u}$ close to $U$. We choose $\lambda=V^{\prime}(\bar{u})$, the equation is then written

$$
\left\{\begin{array}{l}
w^{\prime \prime}=V^{\prime}(w)-V^{\prime}(\bar{u}), \quad y \in \mathbb{R} \\
w^{\prime}(0)=0, w(0)=\bar{u}-\delta
\end{array}\right.
$$


By opposition to the harmonic oscillator, we consider a value $U$ such that the condition (26) holds, which means, using the notation $V^{\prime \prime \prime}=V^{(3)}$,

$$
V^{\prime \prime}(U)<0, \quad V^{(3)}(U) \neq 0, \quad \text { and we set } \omega(U)=\sqrt{-V^{\prime \prime}(U)} .
$$

We have $M^{0}=[U+F(U)] L=\left(1-\frac{d_{1}}{d_{2}}\right) U+V^{\prime}(U)$. We are then reduced to find periodic solutions $w$, with a period $T:=T(\bar{u}, \delta)$ for which a multiple $n \in N^{*}$ gives

$$
n T \sqrt{\varepsilon d_{1}}=L,
$$

and for which the mass conservation gives

$$
n \sqrt{\varepsilon d_{1}}\left[\left(1-\frac{d_{1}}{d_{2}}\right) \int_{0}^{T} w+T V^{\prime}(\bar{u})\right]=M^{0}
$$

and thus

$$
\left(1-\frac{d_{1}}{d_{2}}\right) \frac{1}{T} \int_{0}^{T} w+V^{\prime}(\bar{u})=\frac{M^{0}}{L}
$$

Our claim is that, when $\varepsilon$ varies in an appropriate interval, we can select a one parameter family of values $(\bar{u}(\delta), \delta)$ around $(U, 0)$ where both conditions on the mass $M^{0}$ in $(30)$ and the period $(29)$ are fulfilled.

Expansion of the solution and of the period. The following Lemma holds

Lemma 2.7 Let $\bar{u}$ be an unstable constant steady state and let $T(\bar{u}, \delta)$ be the smallest period of the solution of Equation (27). Assume that $T$ is $\mathcal{C}^{3}$ with respect to the variable $\delta$. Then the following Taylor expansion holds

$$
T(\bar{u}, \delta)=\frac{2 \pi}{\omega}\left(1+\delta^{2} \frac{\left(V^{(3)}\right)^{2}}{24 \omega^{4}}\right)+\mathcal{O}\left(\delta^{3}\right) .
$$

Proof of Lemma 2.7. We first compute an approximation of Equation (27) as follows. We set $z=w-\bar{u}$, and the equation (27) is written

$$
\left\{\begin{array}{l}
z^{\prime \prime}=V^{\prime}(z+\bar{u})-V^{\prime}(\bar{u})=V^{\prime \prime}(\bar{u}) z+\frac{V^{(3)}(\bar{u})}{2} z^{2}+\frac{V^{(4)}(\bar{u})}{6} z^{3}+O\left(\delta^{4}\right) \\
z^{\prime}(0)=0, z(0)=-\delta
\end{array}\right.
$$

We now simplify notations ignoring the dependency of $\bar{u}$ in the formulas. We expand, departing from our knowledge of the first order term, under the form

$$
z=-\delta \cos (\omega y)+\delta^{2} z_{1}+\delta^{3} z_{3}+\mathcal{O}\left(\delta^{4}\right) .
$$

At second order, $z_{1}$ is the solution of

$$
z_{1}^{\prime \prime}=-\omega^{2} z_{1}+\frac{1}{2} V^{(3)} \cos (\omega y)^{2}, \quad z_{1}(0)=z_{1}^{\prime}(0)=0 .
$$

Using the identity $\cos (\omega y)^{2}=\frac{1}{2}+\frac{\cos (2 \omega y)}{2}$, we deduce that

$$
z_{1}^{\prime \prime}=-\omega^{2} z_{1}+\frac{1}{2} V^{(3)}\left(\frac{1}{2}+\frac{\cos (2 \omega y)}{2}\right) .
$$


Setting $z_{2}:=z_{1}-\frac{V^{(3)}}{4 \omega^{2}}$, we obtain

$$
\begin{gathered}
z_{2}^{\prime \prime}=-\omega^{2} z_{2}+\frac{V^{(3)}}{4} \cos (2 \omega y), \quad z_{2}(0)=-\frac{V^{(3)}}{4 \omega^{2}}, z_{2}^{\prime}(0)=0, \\
z_{2}(y)=B_{1} \cos (\omega y)+B_{2} \cos (2 \omega y), \quad B_{1}=-\frac{V^{(3)}}{6 \omega^{2}}, \quad B_{2}=-\frac{V^{(3)}}{12 \omega^{2}} .
\end{gathered}
$$

At third order, $z_{3}$ is the solution of the equation

$$
z_{3}^{\prime \prime}=-\omega^{2} z_{3}-\frac{V^{(3)}}{2} z_{1} \cos (\omega y)-\frac{V^{(4)}}{6} \cos ^{3}(\omega y), \quad z_{3}(0)=z_{3}^{\prime}(0)=0 .
$$

We then deduce that there exists $\alpha, \beta, \gamma$ such that

$$
z_{3}(y)=-\frac{B_{1} V^{(3)}}{4 \omega^{2}}+\alpha \cos (\omega y)+\beta \cos (2 \omega y)+\delta \cos (3 \omega y)
$$

that is

$$
z_{3}(y)=\frac{1}{24}\left(\frac{V^{(3)}}{\omega^{2}}\right)^{2}+\alpha \cos (\omega y)+\beta \cos (2 \omega y)+\delta \cos (3 \omega y) .
$$

We are now able to compute the Taylor expansion of the period $T$ with respect to $\delta$. Integrating Equation (27) between 0 and $T(\delta, \bar{u})$, and to simplify notations we simply use $T$, we deduce that

$$
0=\int_{0}^{T}\left[V^{\prime}(w(y))-V^{\prime}(\bar{u})\right] d y .
$$

We insert the Taylor expansion of $V^{\prime}(w-\bar{u}+\bar{u})$

$$
V^{\prime}(w)-V^{\prime}(\bar{u})=V^{\prime \prime}(\bar{u}) z+\frac{1}{2} V^{(3)}(\bar{u}) z^{2}+\frac{1}{6} V^{(4)}(\bar{u}) z^{3}+\mathcal{O}\left(\delta^{4}\right) .
$$

and thus we arrive at

$$
\begin{aligned}
\mathcal{O}\left(\delta^{4}\right)= & V^{\prime \prime} \int_{0}^{T}\left[-\delta \cos (\omega y)+\delta^{2}\left[B_{1} \cos (\omega y)+B_{2} \cos (2 \omega y)+\frac{V^{(3)}}{4 \omega^{2}}\right]+\delta^{3} z_{3}\right] d y \\
& +\delta^{2} \frac{V^{(3)}}{2} \int_{0}^{T} \cos ^{2}(\omega y) d y+\delta^{3} V^{(3)} \int_{0}^{T} \cos (\omega y) z_{1} d y+\delta^{3} \frac{V^{(4)}}{6} \int_{0}^{T} \cos ^{3}(\omega y) d y .
\end{aligned}
$$

Next, we set

$$
T(\delta)=T(0)+T^{\prime}(0) \delta+T^{\prime \prime}(0) \frac{\delta^{2}}{2}+\mathcal{O}\left(\delta^{3}\right)
$$

and the first order term gives

$$
T(0)=\frac{2 \pi}{\omega}
$$

Next, we use that for all $n \geq 1$

$$
\int_{0}^{T(0)} \cos (n \omega y) d y=0
$$


and we deduce that

$$
\begin{aligned}
\mathcal{O}\left(\delta^{3}\right)=V^{\prime \prime} \int_{0}^{T}[ & \left.-\cos (\omega y)+\delta\left[B_{1} \cos (\omega y)+B_{2} \cos (2 \omega y)+\frac{V^{(3)}}{4 \omega^{2}}\right]+\delta^{2} \frac{1}{24}\left(\frac{V^{(3)}}{\omega^{2}}\right)^{2}\right] d y \\
& +\delta \frac{V^{(3)}}{2} \int_{0}^{T} \cos ^{2}(\omega y) d y \\
= & V^{\prime \prime} \int_{0}^{T}\left[-\cos (\omega y)+\delta\left[B_{1} \cos (\omega y)+B_{2} \cos (2 \omega y)\right]\right] d y+\delta T \frac{V^{\prime \prime} V^{(3)}}{4 \omega^{2}} \\
& +\delta^{2} T(0) \frac{V^{\prime \prime}}{24}\left(\frac{V^{(3)}}{\omega^{2}}\right)^{2}+\delta \frac{V^{(3)}}{2} \int_{0}^{T} \cos ^{2}(\omega y) d y
\end{aligned}
$$

Next, we arrive at

$$
\begin{array}{r}
\mathcal{O}\left(\delta^{3}\right)=V^{\prime \prime} \int_{T(0)}^{T}\left[-\cos (\omega y)+\delta\left[B_{1} \cos (\omega y)+B_{2} \cos (2 \omega y)\right]\right] d y-\delta T \frac{V^{(3)}}{4} \\
-\delta^{2} T(0) \frac{1}{24}\left(\frac{V^{(3)}}{\omega}\right)^{2}+\delta \frac{V^{(3)}}{2}\left[\frac{T(0)}{2}+\int_{T(0)}^{T} \cos ^{2}(\omega y) d y\right]
\end{array}
$$

and because $\cos (\omega y) \approx 1-\frac{\omega(y-T(0))^{2}}{2}$ near $T(0)$, we find

$$
\begin{gathered}
\mathcal{O}\left(\delta^{3}\right)=V^{\prime \prime} \int_{T(0)}^{T}\left[-1+\delta\left[B_{1}+B_{2}\right]\right] d y-\delta T \frac{V^{(3)}}{4} \\
-\delta^{2} T(0) \frac{1}{24}\left(\frac{V^{(3)}}{\omega}\right)^{2}+\delta \frac{V^{(3)}}{2}\left[\frac{T(0)}{2}+\int_{T(0)}^{T} d y\right] \\
=V^{\prime \prime}\left[-\delta T^{\prime}(0)-\frac{\delta^{2}}{2} T^{\prime \prime}(0)+T^{\prime}(0) \delta^{2}\left[B_{1}+B_{2}\right]\right]-\delta^{2} T^{\prime}(0) \frac{V^{(3)}}{4} \\
-\delta^{2} T(0) \frac{1}{24}\left(\frac{V^{(3)}}{\omega}\right)^{2}+\delta^{2} \frac{V^{(3)}}{2} T^{\prime}(0) .
\end{gathered}
$$

The first order term implies that

$$
T^{\prime}(0)=0
$$

and the second order term gives

$$
T^{\prime \prime}(0)=T(0) \frac{\left(V^{(3)}\right)^{2}}{12 \omega^{4}}
$$

This ends the proof of Lemma 2.7.

Mass conservation. We need again to expand the solution $w$ itself. We write

$$
w^{\prime \prime}=-\omega^{2}(w(y)-\bar{u})+O\left(\delta^{2}\right) .
$$


Therefore we can expand

$$
w=\bar{u}-\delta \cos (\omega y)+O\left(\delta^{2}\right)
$$

Integrating the equation (31) between 0 and $\frac{T}{2}$, we find

$$
0=-\omega^{2} \int_{0}^{\frac{T}{2}}[w(y)-\bar{u}] d y+\frac{1}{2} V^{(3)}(\bar{u}) \int_{0}^{\frac{T}{2}}[w(y)-\bar{u}]^{2} d y+O\left(\delta^{3}\right),
$$

that is also written

$$
\begin{aligned}
\omega^{2} \int_{0}^{\frac{T}{2}}[w(y)-\bar{u}] d y & =\frac{\delta^{2}}{2} V^{(3)}(\bar{u}) \int_{0}^{\frac{T}{2}} \cos (\omega y)^{2} d y+O\left(\delta^{3}\right) \\
& =\frac{\delta^{2}}{2} \frac{V^{(3)}(\bar{u})}{\omega} \int_{0}^{\omega \frac{T}{2}} \cos \left(y^{\prime}\right)^{2} d y^{\prime}+O\left(\delta^{3}\right) \\
& =\frac{\delta^{2}}{2} \frac{V^{(3)}(\bar{u})}{\omega} \underbrace{\int_{0}^{\pi} \cos \left(y^{\prime}\right)^{2} d y^{\prime}}_{=\pi / 2}+O\left(\delta^{3}\right) .
\end{aligned}
$$

Therefore the mass condition 30 can be written successively as

$$
\begin{aligned}
& \left(1-\frac{d_{1}}{d_{2}}\right) \frac{2}{T} \int_{0}^{T / 2}(w-\bar{u})+\left[\left(1-\frac{d_{1}}{d_{2}}\right) \bar{u}+V^{\prime}(\bar{u})\right]=\frac{M^{0}}{L}, \\
& \left(1-\frac{d_{1}}{d_{2}}\right) \delta^{2} \frac{V^{(3)}(\bar{u})}{T \omega^{3}} \frac{\pi}{2}+O\left(\delta^{3}\right)+\bar{u}+F(\bar{u})=U+F(U) .
\end{aligned}
$$

Because $u \mapsto u+F(u)$ is locally invertible around $U$, and because $\delta$ is small, this means that we can choose $\bar{u}(\delta)$ according to this expression and get

$$
\bar{u}(\delta)=U-\frac{\delta^{2}}{1+F^{\prime}(U)}\left(1-\frac{d_{1}}{d_{2}}\right) \frac{V^{(3)}(U)}{\omega(U)^{2}} \frac{1}{4}+O\left(\delta^{3}\right) .
$$

Conclusion According to Lemma 2.7, the intervals of values $\epsilon$ are finally given by

$$
\begin{aligned}
\sqrt{\varepsilon d_{1}} & =\frac{L}{n} \frac{\omega(\bar{u})}{2 \pi}\left[1+\frac{\delta^{2}\left(V^{(3)}(\bar{u})\right)^{2}}{24 \omega(\bar{u})^{4}}+O\left(\delta^{3}\right)\right] \\
& =\frac{L}{n} \frac{\omega(U)}{2 \pi}\left[1+\delta^{2} \frac{\left(V^{(3)}(U)\right)^{2}}{(\omega(U))^{2}}\left(\frac{1}{24(\omega(U))^{2}}+\frac{L\left(1-\frac{d_{1}}{d_{2}}\right)}{16 n \pi\left(1+F^{\prime}(U)\right) \omega(U)}\right)+O\left(\delta^{3}\right)\right] .
\end{aligned}
$$

This ends the proof of Theorem 2.6.

\subsection{Interpretation via entropy functionals}

In this part, we again consider equation on a regular bounded domain $\Omega \subset \mathbb{R}^{d}$

$$
\left\{\begin{array}{l}
\partial_{t} u_{\varepsilon}-d_{1} \Delta u_{\varepsilon}=\varepsilon^{-1}\left(v_{\varepsilon}-F\left(u_{\varepsilon}\right)\right) \\
\partial_{t} v_{\varepsilon}-d_{2} \Delta v_{\varepsilon}=-\varepsilon^{-1}\left(v_{\varepsilon}-F\left(u_{\varepsilon}\right)\right) .
\end{array}\right.
$$


Following [17], several entropy functionals allow to tackle, on the one hand the asymptotic dynamic of the solution and on the other hand the fast reaction limit, in the case where no Turing instabilities may appear. More precisely, we have the following three keys equality

Let $G$ be an antiderivative of $F$. Multiplying the equation on $u_{\varepsilon}$ by $F\left(u_{\varepsilon}\right)$ and on $v_{\varepsilon}$ by $v_{\varepsilon}$, we have

$$
\begin{aligned}
\frac{d}{d t} \int_{\Omega}\left(G\left(u_{\varepsilon}\right)(x, t)+\frac{1}{2} v_{\varepsilon}^{2}(x, t)\right) d x= & -d_{1} \int_{\Omega} F^{\prime}\left(u_{\varepsilon}\right)\left|\nabla u_{\varepsilon}\right|^{2} d x-d_{2} \int_{\Omega}\left|\nabla v_{\varepsilon}\right|^{2} d x \\
& -\frac{1}{\varepsilon} \int_{\Omega}\left(v_{\varepsilon}-F\left(u_{\varepsilon}\right)\right)^{2} d x .
\end{aligned}
$$

Multiplying the equation on $w_{\varepsilon}=u_{\varepsilon}+v_{\varepsilon}$ by $w_{\varepsilon}$, we find that

$$
\frac{d}{d t} \int_{\Omega} \frac{w_{\varepsilon}^{2}}{2} d x=-\int_{\Omega} \nabla w_{\varepsilon} \cdot\left(d_{1} \nabla u_{\varepsilon}+d_{2} \nabla v_{\varepsilon}\right) d x
$$

Multiplying the equation on $u_{\varepsilon}$ by $\Delta u_{\varepsilon}$, we find that

$$
\frac{d}{d t} \int_{\Omega} \frac{\left|\nabla u_{\varepsilon}\right|^{2}}{2} d x=-d_{1} \int_{\Omega}\left(\Delta u_{\varepsilon}\right)^{2} d x+\frac{1}{\varepsilon}\left(\int_{\Omega} \nabla u_{\varepsilon} \cdot \nabla v_{\varepsilon} d x-\int_{\Omega} F^{\prime}\left(u_{\varepsilon}\right)\left|\nabla u_{\varepsilon}\right|^{2} d x\right)
$$

and that

$$
\frac{d}{d t} \int_{\Omega} \frac{\left|\nabla u_{\varepsilon}\right|^{2}}{2} d x=-d_{1} \int_{\Omega}\left(\Delta u_{\varepsilon}\right)^{2} d x-\frac{1}{\varepsilon}\left(\int_{\Omega}\left(v_{\varepsilon}-F\left(u_{\varepsilon}\right)\right) \Delta u_{\varepsilon} d x\right) .
$$

From this, we deduce the following proposition, taken from [17] (see also Appendix B, for more details)

Proposition 2.8 Combining equalities (32)-(35), the following equality holds

$$
\begin{array}{ll}
\frac{d}{d t} & \int_{\Omega}\left(G\left(u_{\varepsilon}\right)(x, t)+\frac{1}{2} v_{\varepsilon}^{2}(x, t)+\frac{\varepsilon d_{1}}{2}\left|\nabla u_{\varepsilon}\right|^{2}+\frac{\varepsilon d_{1}^{2}}{4\left(d_{2}-d_{1}\right)} w_{\varepsilon}^{2}\right) d x= \\
& -\varepsilon \int_{\Omega}\left(d_{1} \Delta u_{\varepsilon}+\frac{1}{\varepsilon}\left(v_{\varepsilon}-F\left(u_{\varepsilon}\right)\right)\right)^{2} d x-\frac{1}{d_{2}-d_{1}} \int_{\Omega}\left(d_{1} \nabla u_{\varepsilon}+d_{2} \nabla v_{\varepsilon}\right)^{2} d x .
\end{array}
$$

Combining equalities (32) and (33), the following equality holds for $d_{2}>d_{1}$

$$
\begin{aligned}
\frac{d}{d t} \int_{\Omega}\left(G\left(u_{\varepsilon}\right)(x, t)+\right. & \left.\frac{1}{2} v_{\varepsilon}^{2}(x, t)+\frac{d_{1}}{d_{2}-d_{1}} w_{\varepsilon}^{2}\right) d x=-d_{1} \int_{\Omega}\left(F^{\prime}\left(u_{\varepsilon}\right)+\frac{d_{1}}{d_{2}}\right)\left|\nabla u_{\varepsilon}\right|^{2} d x \\
& -\frac{d_{1}+d_{2}}{d_{2}\left(d_{2}-d_{1}\right)} \int_{\Omega}\left(d_{1} \nabla u_{\varepsilon}+d_{2} \nabla v_{\varepsilon}\right)^{2} d x-\frac{1}{\varepsilon} \int_{\Omega}\left(v_{\varepsilon}-F\left(u_{\varepsilon}\right)\right)^{2} d x
\end{aligned}
$$

and for $d_{1}>d_{2}$

$$
\begin{aligned}
\frac{d}{d t} \int_{\Omega}\left(G\left(u_{\varepsilon}\right)(x, t)+\right. & \left.\frac{1}{2} v_{\varepsilon}^{2}(x, t)+\frac{d_{2}}{d_{1}-d_{2}} w_{\varepsilon}^{2}\right) d x=-d_{1} \int_{\Omega}\left(F^{\prime}\left(u_{\varepsilon}\right)+\frac{d_{2}}{d_{1}}\right)\left|\nabla u_{\varepsilon}\right|^{2} d x \\
& -\frac{d_{1}+d_{2}}{d_{2}\left(d_{1}-d_{2}\right)} \int_{\Omega}\left(d_{1} \nabla u_{\varepsilon}+d_{2} \nabla v_{\varepsilon}\right)^{2} d x-\frac{1}{\varepsilon} \int_{\Omega}\left(v_{\varepsilon}-F\left(u_{\varepsilon}\right)\right)^{2} d x
\end{aligned}
$$

These inequalities have standard consequences in term of behaviors of solutions that we recall and complete with a larger range of validity for the parameters. 
Long time asymptotic, $\varepsilon>0$ fixed. Using the energy dissipation, the equality (36) ensures that, asymptotically, as $t \rightarrow \infty$, the solutions converge to a stationary state. We refer to [15, 16] for more precise results on this topic.

The limit $\varepsilon \rightarrow 0, d_{1} \neq d_{2}$. The equalities (37), (38) are useful in the two cases when $d_{2}>d_{1}$ and $\frac{d_{1}}{d_{2}}+F^{\prime}(\cdot) \geq c_{o}>0$ or $d_{1}>d_{2}$ and $\frac{d_{2}}{d_{1}}+F^{\prime}(\cdot) \geq c_{o}>0$. On the one hand, it shows that the solution necessary converges in long time to a constant stationary state in accordance with Theorem 2.4

On the other hand, these equalities provide us with uniform bounds, with respect to $\varepsilon$, on the derivatives of $u$ and $v$. They give the existence of a constant $C>0$ independent of $\varepsilon>0$ such that

$$
\int_{t=0}^{+\infty} \int_{\Omega}\left|\nabla u_{\varepsilon}\right|^{2}+\left|\nabla v_{\varepsilon}\right|^{2} d x d t<C
$$

The situation is exactly the same as in [4] which treats the case $F^{\prime}(\cdot) \geq 0$. Therefore, we can extend the result in [4, with the same proof, to obtain the

Theorem 2.9 Assume $d_{1}<d_{2}$ and $\frac{d_{1}}{d_{2}}+F^{\prime}(\cdot) \geq c_{o}>0$, or $d_{1}>d_{2}$ and $\frac{d_{2}}{d_{1}}+F^{\prime}(\cdot) \geq c_{o}>0$. As $\varepsilon \rightarrow 0, w_{\varepsilon}=u_{\varepsilon}+v_{\varepsilon}$ converges a.e. to a function $w$ that satisfies the equation

$$
\left\{\begin{array}{l}
\partial_{t} w-\Delta A(w)=0, \quad x \in \Omega, t \geq 0 \\
\frac{\partial w}{\partial \nu}=0 \quad \text { on } \partial \Omega,
\end{array}\right.
$$

where $A$ is defined in Section 1.1 and is written

$$
A(w):=d_{1} u+d_{2} F(u), \text { with } w=u+F(u) .
$$

Let us mention that the question of convergence of $u_{\varepsilon}, v_{\varepsilon}$, when $\varepsilon \rightarrow 0$, in the case where (20) is not satisfied, is very difficult to tackle because of the oscillations of size $\frac{1}{\sqrt{d_{1} \varepsilon}}$ that may appear as shown in Section 2.2.

The case $d_{1}=d_{2}$ is simpler but requires a specific proof because $w_{\varepsilon}$ satisfies the heat equation.

\section{A $2 \times 2$ cross diffusion system}

In Section 2, in the context of a system of two reaction-diffusion equations, we established an equivalence between Turing instability for a steady state of (1), and ill-posedeness (e.g. "backward parabolicity") of the corresponding fast reaction limit equation. Here, we aim at exhibiting a class of $4 \times 4$ reaction-diffusion systems for which the asymptotic $\varepsilon \rightarrow 0$ produces a full $2 \times 2$ cross diffusion systems and in which the equivalence Turing instability / backward parabolicity holds as before.

We use the notations and assumptions of section 1.2 .

\subsection{Turing instabilities and backward cross-diffusion equation}

Here again, we obtain the direct analog of the "backward parabolicity" property for the cross diffusion system (8), that is the negativity of the Jacobian of the matrix $M$ defined below. More precisely we have the following result 
Proposition 3.1 Assume (14). Then, a linearly constant steady state $\bar{U}={ }^{t}\left(\bar{u}^{1}, \bar{u}^{2}, \bar{u}^{3}, \bar{u}^{4}\right)$ is asymptotically Turing unstable for (6) if and only if $\operatorname{det} M(\bar{V})<0$, where

$$
M=\left(\begin{array}{ll}
A_{v} & A_{w} \\
B_{v} & B_{w}
\end{array}\right), \quad \bar{V}:=\frac{1}{2}\left(\begin{array}{c}
\bar{u}^{1}+\bar{u}^{2} \\
\bar{u}^{3}+\bar{u}^{4}
\end{array}\right) .
$$

In other words, Turing instability is equivalent to backward parabolicity of the cross-diffusion system.

We recall that, because we assume self-diffusion is positive in (14), that means $A_{v}>0, B_{w}>0$, the matrix $M$ has always an eigenvalue with positive real part; the cross-diffusion system cannot be fully backward with two negative eigenvalues.

Proof of Proposition 3.1. We define the change of variables

$$
v_{\varepsilon}=\frac{u_{\varepsilon}^{1}+u_{\varepsilon}^{2}}{2}, \quad w_{\varepsilon}=\frac{u_{\varepsilon}^{3}+u_{\varepsilon}^{4}}{2}, \quad y_{\varepsilon}=\frac{d_{1} u_{\varepsilon}^{1}+d_{2} u_{\varepsilon}^{2}}{2}, \quad z_{\varepsilon}=\frac{d_{3} u_{\varepsilon}^{3}+d_{4} u_{\varepsilon}^{4}}{2} .
$$

We find that

$$
u_{\varepsilon}^{1}=2 \frac{d_{2} v_{\varepsilon}-y_{\varepsilon}}{d_{2}-d_{1}} \text { and } u^{3}=2 \frac{d_{4} w_{\varepsilon}-z_{\varepsilon}}{d_{4}-d_{3}} .
$$

With the new variables, we obtain the following system of equations

$$
\left\{\begin{array}{l}
\partial_{t} y_{\varepsilon}=\left(d_{2}+d_{1}\right) \Delta y_{\varepsilon}-d_{1} \Delta v_{\varepsilon}+\frac{\left(d_{1}-d_{2}\right)}{2 \varepsilon}\left(R\left(v_{\varepsilon}, w_{\varepsilon}\right)-v_{\varepsilon} \frac{\left(d_{1}+d_{2}\right)}{\left(d_{2}-d_{1}\right)}+\frac{2 y_{\varepsilon}}{\left(d_{2}-d_{1}\right)}\right), \\
\partial_{t} v_{\varepsilon}=\Delta y_{\varepsilon}, \\
\partial_{t} z_{\varepsilon}=\left(d_{4}+d_{3}\right) \Delta z_{\varepsilon}-d_{3} \Delta w_{\varepsilon}+\frac{\left(d_{3}-d_{4}\right)}{2 \varepsilon}\left(S\left(v_{\varepsilon}, w_{\varepsilon}\right)-w_{\varepsilon} \frac{\left(d_{3}+d_{4}\right)}{\left(d_{4}-d_{3}\right)}+\frac{2 z_{\varepsilon}}{\left(d_{4}-d_{3}\right)}\right), \\
\partial_{t} w_{\varepsilon}=\Delta z_{\varepsilon},
\end{array}\right.
$$

and so

$$
\left\{\begin{array}{l}
\partial_{t} y_{\varepsilon}=\left(d_{2}+d_{1}\right) \Delta y_{\varepsilon}-d_{1} \Delta v_{\varepsilon}+\frac{1}{\varepsilon}\left(A(v, w)-y_{\varepsilon}\right) \\
\partial_{t} v_{\varepsilon}=\Delta y_{\varepsilon} \\
\partial_{t} z_{\varepsilon}=\left(d_{4}+d_{3}\right) \Delta z_{\varepsilon}-d_{3} \Delta w_{\varepsilon}+\frac{1}{\varepsilon}\left(B(v, w)-z_{\varepsilon}\right) \\
\partial_{t} w_{\varepsilon}=\Delta z_{\varepsilon}
\end{array}\right.
$$

The linearized equation, around a stationary state $(\bar{v}, \bar{w}, \bar{y}, \bar{z})$, is given by

$$
\left\{\begin{array}{l}
\partial_{t} y_{\varepsilon}=\left(d_{2}+d_{1}\right) \Delta y_{\varepsilon}-d_{1} \Delta v_{\varepsilon}+\frac{1}{\varepsilon}\left(A_{v}(\bar{v}, \bar{w}) v_{\varepsilon}+A_{w}(\bar{v}, \bar{w}) w_{\varepsilon}\right)-\frac{y_{\varepsilon}}{\varepsilon}, \\
\partial_{t} v_{\varepsilon}=\Delta y_{\varepsilon} \\
\partial_{t} z_{\varepsilon}=\left(d_{4}+d_{3}\right) \Delta z_{\varepsilon}-d_{3} \Delta w_{\varepsilon}+\frac{1}{\varepsilon}\left(B_{v}(\bar{v}, \bar{w}) v_{\varepsilon}+B_{w}(\bar{v}, \bar{w}) w_{\varepsilon}\right)-\frac{z_{\varepsilon}}{\varepsilon} \\
\partial_{t} w_{\varepsilon}=\Delta z_{\varepsilon}
\end{array}\right.
$$

Now, we decompose each solution of 40 with respect to the eigenfunctions $E_{i}$ of the Laplacian associated to the eigenvalues $\lambda_{i} \leq 0$, that is we write

$$
v_{\varepsilon}=\sum_{i=0}^{+\infty} E_{i} v_{\varepsilon}^{i}(t), \quad w_{\varepsilon}=\sum_{i=0}^{+\infty} E_{i} w_{\varepsilon}^{i}(t), \quad y_{\varepsilon}=\sum_{i=0}^{+\infty} E_{i} y_{\varepsilon}^{i}(t), \quad z_{\varepsilon}=\sum_{i=0}^{+\infty} E_{i} z_{\varepsilon}^{i}(t) .
$$

We find that

$$
\frac{d}{d t}\left(\begin{array}{c}
v_{\varepsilon}^{i} \\
w_{\varepsilon}^{i} \\
y_{\varepsilon}^{i} \\
z_{\varepsilon}^{i}
\end{array}\right)=M_{i}\left(\begin{array}{c}
v_{\varepsilon}^{i} \\
w_{\varepsilon}^{i} \\
y_{\varepsilon}^{i} \\
z_{\varepsilon}^{i}
\end{array}\right), \quad M_{i}=\left(\begin{array}{cccc}
0 & 0 & \lambda_{i} & 0 \\
0 & 0 & 0 & \lambda_{i} \\
a^{31} & a^{32} & a^{33} & 0 \\
a^{41} & a^{42} & 0 & a^{44}
\end{array}\right)
$$


with

$$
\begin{aligned}
& a^{31}=-d_{1} \lambda_{i}+\frac{1}{\varepsilon} A_{v}, \quad a^{32}=\frac{1}{\varepsilon} A_{w}, \quad a^{33}=\left(d_{1}+d_{2}\right) \lambda_{i}-\frac{1}{\varepsilon}, \\
& a^{41}=\frac{1}{\varepsilon} B_{v}, \quad a^{42}=-d_{3} \lambda_{i}+\frac{1}{\varepsilon} B_{w}, \quad a^{44}=\left(d_{3}+d_{4}\right) \lambda_{i}-\frac{1}{\varepsilon} .
\end{aligned}
$$

Let us mention that, because $\lambda_{i}<0, A_{v}>0, B_{w}>0$ we have

$$
a^{31}>0, \quad a^{33}<0, \quad a^{42}>0, \quad a^{44}<0 .
$$

We find that

$$
\begin{aligned}
& P(\eta):=\operatorname{Det}\left(A_{i}-\eta I_{4}\right) \\
& =\eta^{4}+\eta^{3}\left(-a^{33}-a^{44}\right)+\eta^{2}\left(a^{33} a^{44}-\lambda_{i}\left(a^{42}+a^{31}\right)\right)+\eta \lambda_{i}\left(a^{31} a^{44}+a^{42} a^{33}\right)+\lambda_{i}^{2}\left(a^{31} a^{42}-a^{41} a^{32}\right) .
\end{aligned}
$$

We have

$$
a^{31} a^{42}-a^{41} a^{32}<0 \Leftrightarrow\left(A_{v} B_{w}-B_{v} A_{w}\right)<0 \quad \text { if } \varepsilon \text { small enough },
$$

hence, we obtain for $\varepsilon>0$ small enough, that $P$ has a positive root if and only if $\left(A_{v} B_{w}-B_{v} A_{w}\right)<0$ which ends the proof of Proposition 3.1 .

\subsection{Entropy functional}

In general, we could not find an entropy functional for the system (6). However, when there exists two functions $\phi_{1}$ and $\phi_{2}$ such that

$$
\phi_{1}(w)+\int_{0}^{v} A(y, w) d y=\phi_{2}(v)+\int_{0}^{w} B(v, y) d y:=\Phi(v, w)
$$

the following proposition holds

Proposition 3.2 Assume that assumption (41) holds. Let

$$
E_{1}(t):=\frac{1}{2}\left(\|\nabla y\|_{L^{2}(\Omega)}^{2}+\|\nabla z\|_{L^{2}(\Omega)}^{2}+d_{1}\|\nabla v\|_{L^{2}(\Omega)}^{2}+d_{3}\|\nabla w\|_{L^{2}(\Omega)}^{2}\right)+\frac{1}{\varepsilon} \int_{\Omega} \Phi(t, x) d x .
$$

Then, the following estimate holds

$$
\frac{d}{d t} E_{1}(t)=-\frac{1}{\varepsilon}\left(\|\nabla y\|_{L^{2}(\Omega)}^{2}+\|\nabla z\|_{L^{2}(\Omega)}^{2}\right)-\left(d_{1}+d_{2}\right)\|\Delta y\|_{L^{2}(\Omega)}^{2}-\left(d_{3}+d_{4}\right)\|\Delta z\|_{L^{2}(\Omega)}^{2} .
$$

Proof of Proposition 3.2. We work on the system (40). Multiplying the equation on $y_{\varepsilon}$ by $\Delta y_{\varepsilon}$ and the equation on $z_{\varepsilon}$ by $\Delta z_{\varepsilon}$, and the equations on $v$ and $w$ by $\Phi$, we find that

$$
\begin{aligned}
\frac{d}{d t} \quad & \left(\frac{1}{2}\left(\|\nabla y\|_{L^{2}(\Omega)}^{2}+\|\nabla z\|_{L^{2}(\Omega)}^{2}+d_{1}\|\nabla v\|_{L^{2}(\Omega)}^{2}+d_{3}\|\nabla w\|_{L^{2}(\Omega)}^{2}\right)+\frac{1}{\varepsilon} \int_{\Omega} \Phi(t, x) d x\right) \\
& =-\frac{1}{\varepsilon}\left(\|\nabla y\|_{L^{2}(\Omega)}^{2}+\|\nabla z\|_{L^{2}(\Omega)}^{2}\right)-\left(d_{1}+d_{2}\right)\|\Delta y\|_{L^{2}(\Omega)}^{2}-\left(d_{3}+d_{4}\right)\|\Delta z\|_{L^{2}(\Omega)}^{2} .
\end{aligned}
$$




\section{A Hamiltonian system}

We give another approach to the existence of periodic solutions in the context of Theorem 2.6 .

We consider $\mu \in \mathscr{C}^{1}(\mathbb{R})$ and the vector field $\Theta: \mathbb{R}^{2} \rightarrow \mathbb{R}^{2}$

$$
\Theta\left(\begin{array}{l}
y_{1} \\
y_{2}
\end{array}\right)=\left(\begin{array}{c}
y_{2} \\
\mu\left(y_{1}\right)
\end{array}\right)
$$

We assume appropriate growth conditions on $\mu$ so that, for any $Y_{0} \in \mathbb{R}^{2}$ the system

$$
\begin{aligned}
& Y^{\prime}(t)=\Theta(Y(t)), \\
& Y(0)=Y_{0}
\end{aligned}
$$

admits a global solution. We denote by $\Phi_{t}\left(Y_{0}\right)$ the corresponding flow.

Proposition A.1 If $\mu^{\prime}(\bar{u})<0=\mu(\bar{u})$ for some real number $\bar{u}$, there exists a neighborhood $V$ of $(\bar{u}, 0)$ such that for any $Y_{0} \in V \backslash(\bar{u}, 0), t \mapsto \Phi_{t}\left(Y_{0}\right)$ is periodic and non constant.

Proof. By assumption the point $\bar{Y}:=(\bar{u}, 0)$ is an equilibrium for our system. A straightforward computation shows that if $\gamma^{\prime}=\mu$ and $t \mapsto\left(y_{1}(t), y_{2}(t)\right)$ is a solution, then the following function is constant

$$
t \mapsto \frac{y_{2}(t)^{2}}{2}-\gamma\left(y_{1}(t)\right)
$$

The corresponding Hamiltonian $H$ satisfies $\nabla H\left(y_{1}, y_{2}\right)=\left(-\mu\left(y_{1}\right), y_{2}\right)$ and

$$
\mathrm{D}^{2} H\left(y_{1}, y_{2}\right)=\left(\begin{array}{cc}
-\mu^{\prime}\left(y_{1}\right) & 0 \\
0 & 1
\end{array}\right)
$$

In particular $\nabla H(\bar{Y})=0$ and $\mathrm{D}^{2} H(\bar{Y})>0: H$ has a local strict minimum at $\bar{Y}$. Take $W$ a neighborhood of $\bar{Y}$ on which $Y \neq \bar{Y} \Rightarrow H(Y)>H(\bar{Y})$ and also $\mu\left(y_{1}\right)\left(y_{1}-\bar{u}\right)<0$, the latter being possible thanks to the assumption $\mu^{\prime}(\bar{u})<0=\mu(\bar{u})$. Consider a closed circle $\mathscr{C} \subset W$ around $\bar{Y}$. If $\delta=\min _{\mathscr{C}} H$, by continuity we have the existence of a neighborhood $V$ of $\bar{Y}$ on which $\sup _{V} H<\delta$. For any $Y_{0} \in V$ the map $t \mapsto H\left(\Phi_{t}\left(Y_{0}\right)\right)$ is constant, in particular $\Phi_{t}\left(Y_{0}\right)$ may not cross $\mathscr{C}$. If $D$ is the open disk delimited by $\mathscr{C}$ we have just proved $Y_{0} \in V \Rightarrow\left\{\Phi_{t}\left(Y_{0}\right): t \geq 0\right\} \subset D$.

Now if $Y_{0} \in V$ and $\Phi_{t}\left(Y_{0}\right)=\left(y_{1}(t), y_{2}(t)\right)$, it is not possible to have $y_{1}>\bar{u}$ near $+\infty$. Indeed, recall that near $+\infty$ a bounded concave nonincreasing function is constant. In particular, if $y_{1}>\bar{u}$ near $+\infty$, then $y_{1}^{\prime \prime}=y_{2}=\mu\left(y_{1}\right)<0$ near infinity, so that $y_{1}$ is concave and non constant ; since $\left\{\Phi_{t}\left(Y_{0}\right): t \geq 0\right\} \subset D, y_{1}$ must be increasing. But then the same argument applies on $y_{1}^{\prime}$ which is nonincreasing (because $y_{1}^{\prime \prime}<0$ ) and concave (because $y_{1}^{\prime \prime}=\mu\left(y_{1}\right)$ is decreasing). In a similar way, we cannot have $y_{1}(t)<\bar{u}$ near $+\infty$.

We eventually proved that $t \mapsto y_{1}(t)$ takes the value $\bar{u}$ infinitely many times. But because of the Hamiltonian equation, for a given value of $y_{1}(t)$, there are at most two possible choices for $y_{2}(t)$ : the map $t \mapsto\left(y_{1}(t), y_{2}(t)\right)$ may not be injective and is hence periodic. Since $\Theta$ vanishes only at $\bar{Y}$ on $V$, $t \mapsto \Phi_{t}\left(Y_{0}\right)$ is non constant if $Y_{0} \neq \bar{Y}$.

Proposition A.2 Under the Assumptions of Proposition A.1, for a possibly smaller neighborhood $V$, the following holds: for any $z_{0}<\bar{u}$ such that $\left(z_{0}, 0\right) \in V$, the period function $T\left(z_{0}\right)$ is well-defined. 
Proof. Fix $\gamma$ such that $\gamma^{\prime}=\mu$ and $\gamma(\bar{\mu})=0$. Then, $\gamma$ is strictly increasing before $\bar{\mu}\left(\gamma_{\uparrow}\right.$ the restriction) and strictly decreasing after $\left(\gamma_{\downarrow}\right.$ the restriction). In particular, taking $V$ smaller if necessary, we can assume that for any $z_{0}<\bar{\mu}$ such that $\left(z_{0}, 0\right) \in V$, there exists $h\left(z_{0}\right)>\bar{\mu}$ such that $\gamma\left(z_{0}\right)=\gamma\left(h\left(z_{0}\right)\right)$. The function $h$ is actually defined by the formula $h\left(z_{0}\right)=\gamma_{\downarrow}^{-1}\left(\gamma\left(z_{0}\right)\right)$ and is $\mathscr{C}^{1}$. As noticed before, along the flow the map $\left(y_{1}, y_{2}\right) \mapsto y_{2}^{2} / 2-\gamma\left(y_{1}\right)$ is constant, in particular starting from $\left(z_{0}, 0\right)$, the value of the hamiltonian remains equal to $H_{0}:=-\gamma\left(z_{0}\right)$. But the only other point of the real axis (belonging to $V$ ) on which the hamiltonian can take the same value is $\left(h\left(z_{0}\right), 0\right)$. Since the phase portrait is symmetric w.r.t. to the real axis, the period function is given by $T\left(z_{0}\right):=2 \min \{t>0$ : $\left.\gamma\left(y_{1}(t)\right)=-H_{0}\right\}$. On the interval $\left(0, T\left(z_{0}\right) / 2\right)$ the map $t \mapsto y_{1}(t)$ is strictly increasing so that we infer (using $s=y_{1}(t)$ )

$$
\frac{T\left(z_{0}\right)}{2}=\int_{0}^{\frac{T\left(z_{0}\right)}{2}} d t=\int_{y_{1}(0)}^{y_{1}\left(\frac{T\left(z_{0}\right)}{2}\right)} \frac{d s}{y_{1}^{\prime}\left(y_{1}^{-1}(s)\right)}=\int_{z_{0}}^{h\left(z_{0}\right)} \frac{d s}{y_{2}\left(y_{1}^{-1}(s)\right)} .
$$

Using the constraint $y_{2}(t)^{2} / 2-\gamma\left(y_{1}(t)\right)=H_{0}$, we get here

$$
\frac{T\left(z_{0}\right)}{2}=\frac{1}{\sqrt{2}} \int_{z_{0}}^{h\left(z_{0}\right)} \frac{d s}{\sqrt{H_{0}+\gamma(s)}} .
$$

\section{B The entropy}

We explain why two specific idendities are singled out in section 2.4. We set

$$
\alpha=\frac{d_{1}+d_{2}}{\left|d_{2}-d_{1}\right|}-1
$$

and define

$$
\begin{gathered}
I(t)=\int_{\Omega}\left(G\left(u_{\varepsilon}\right)(x, t)+\frac{1}{2} v_{\varepsilon}^{2}(x, t)+\alpha \frac{w_{\varepsilon}^{2}}{2}\right) d x, \\
J(t)=\frac{1}{\varepsilon} \int_{\Omega}\left(v_{\varepsilon}-F\left(u_{\varepsilon}\right)\right)^{2} d x .
\end{gathered}
$$

We compute, combining the idendities $(32)$ and 33

$$
\begin{aligned}
\frac{d I(t)}{d t}+J(t)= & -d_{1} \int_{\Omega} F^{\prime}\left(u_{\varepsilon}\right)\left|\nabla u_{\varepsilon}\right|^{2} d x-d_{2} \int_{\Omega}\left|\nabla v_{\varepsilon}\right|^{2} d x-\alpha \int_{\Omega} \nabla w_{\varepsilon} \cdot\left(d_{1} \nabla u_{\varepsilon}+d_{2} \nabla v_{\varepsilon}\right) d x \\
= & -d_{1} \int_{\Omega}\left[F^{\prime}\left(u_{\varepsilon}\right)+\beta\right]\left|\nabla u_{\varepsilon}\right|^{2} d x \\
& \quad-\int_{\Omega}\left[d_{2}(1+\alpha)\left|\nabla v_{\varepsilon}\right|^{2}+\alpha\left(d_{1}+d_{2}\right) \nabla u_{\varepsilon} \cdot \nabla v_{\varepsilon}+d_{1}(\alpha-\beta)\left|\nabla u_{\varepsilon}\right|^{2}\right] d x
\end{aligned}
$$

Depending on our choice of $\alpha$, we find $\beta$ such that the last expression is a square, that is

$$
\begin{gathered}
\alpha^{2}\left(d_{1}+d_{2}\right)^{2}=4 d_{2}(1+\alpha) d_{1}(\alpha-\beta), \\
-\alpha^{2}\left(d_{1}-d_{2}\right)^{2}+4 d_{1} d_{2} \alpha=4 d_{1} d_{2}(\alpha+1) \beta .
\end{gathered}
$$


And thus the largest value of $\beta$ is given by

$$
4 d_{1} d_{2} \beta=\max _{\alpha>0} \frac{-\alpha^{2}\left(d_{1}-d_{2}\right)^{2}+4 d_{1} d_{2} \alpha}{\alpha+1}
$$

the first order condition gives

$$
\begin{gathered}
\alpha^{2}\left(d_{1}-d_{2}\right)^{2}+2 \alpha\left(d_{1}-d_{2}\right)^{2}-4 d_{1} d_{2}=0 \\
\left(d_{1}-d_{2}\right)^{2}(\alpha+1)^{2}=\left(d_{1}-d_{2}\right)^{2}+4 d_{1} d_{2}=\left(d_{1}+d_{2}\right)^{2}
\end{gathered}
$$

and the positive root is the value in (42).

- For $d_{2}>d_{1}$, we find

$$
\alpha=\frac{2 d_{1}}{d_{2}-d_{1}}, \quad \beta=\frac{d_{1}}{d_{2}},
$$

because $4 d_{1} d_{2} \beta=\frac{2 \alpha\left(d_{1}-d_{2}\right)^{2}-4 d_{1} d_{2}+4 d_{1} d_{2} \alpha}{\alpha+1}=\frac{2 \alpha\left(d_{1}^{2}+d_{2}^{2}\right)-4 d_{1} d_{2}}{\alpha+1}=\frac{1}{d_{1}+d_{2}}\left[4 d_{1}\left(d_{2}^{2}+d_{1}^{2}\right)-4 d_{1} d_{2}\left(d_{2}-d_{1}\right)\right]$.

- For $d_{1}>d_{2}$, we find

$$
\alpha=\frac{2 d_{2}}{d_{1}-d_{2}}, \quad \beta=\frac{d_{2}}{d_{1}},
$$

\section{References}

[1] M. Bendahmane, T. Lepoutre, A. Marrocco, and B. Perthame, Conservative cross diffusions and pattern formation through relaxation, J. Math. Pures Appl.,, 92 (2009), pp. 651667 .

[2] M. Bertsch, F. Smarrazzo, And A. Tesei, Pseudo-parabolic regularization of forwardbackward parabolic equations: power-type nonlinearities, J. Reine Angew. Math., 712 (2016), pp. 51-80.

[3] S. Bolotin and R. S. MacKay, Isochronous potential, in Proceedings of the third conference: Localization and Energy Transfer in Nonlinear Systems, L. Vázquez, R. S. MacKay, and M. P. Zorzano, eds., London 2003, pp. 217-224.

[4] D. Bothe And D. Hilhorst, A reaction-diffusion system with fast reversible reaction, Journal of Mathematical Analysis and Applications, 286 (2003), pp. 125 - 135.

[5] D. Bothe, M. Pierre, And G. Rolland, Cross-diffusion limit for a reaction-diffusion system with fast reversible reaction, Communications in Partial Differential Equations, 37 (2012), pp. 1940-1966.

[6] J. A. Carillo, S. Hittmeier, And A. Jüngel, Cross diffusion and nonlinear diffusion preventing blow-up in the keller-segel model, Mathematical Models and Methods in Applied Sciences, 22 (2012), p. 1250041.

[7] L. Desvillettes And A. TRescases, New results for triangular reaction cross diffusion system, Journal of Mathematical Analysis and Applications, 430 (2015), pp. 32-59.

[8] L. C. Evans And M. Portilheiro, Irreversibility and hysteresis for a forward-backward diffusion equation, Math. Models Methods Appl. Sci., 14 (2004), pp. 1599-1620. 
[9] C. A. Haselwandter, M. Kardar, A. Triller, and R. A. Da Silveira, Self-assembly and plasticity of synaptic domains through a reaction-diffusion mechanism, Phys. Rev. E, 92 (2015), p. 032705.

[10] M. Iida, M. Mimura, And H. Ninomiya, Diffusion, cross-diffusion and competitive interaction, J. Math. Biol., 53 (2006), pp. 617-641.

[11] D. Iron And J. Rumsey, A model of cell surface receptor aggregation, J. Math. Biol., 75 (2017), pp. 705-731.

[12] S. Ishihara, M. Otsuji, And A. Mochizuki, Transient and steady state of mass-conserved reaction-diffusion systems, Phys. Rev. E, 75 (2007).

[13] H. Izuhara And M. Mimura, Reaction-diffusion system approximation to the cross-diffusion competition system, Hiroshima Math. J., 38 (2008), pp. 315-347.

[14] P. LAfitTe And C. MAscia, Numerical exploration of a forward-backward diffusion equation, Math. Models Methods Appl. Sci., 22 (2012), pp. 1250004, 33.

[15] Y. MORITA, Spectrum comparison for a conserved reaction-diffusion system with a variational property, J. Appl. Anal. Comput., 2 (2012), pp. 57-71.

[16] Y. Morita And T. OGaWA, Stability and bifurcation of nonconstant solutions to a reactiondiffusion system with conservation of mass, Nonlinearity, 23 (2010), pp. 1387-1411.

[17] Y. Morita AND N. Shinjo, Reaction-diffusion models with a conservation law and pattern formations, Josai Mathematical Monographs, 9 (2016), pp. 177-190.

[18] A. Novick-Cohen and R. L. Pego, Stable patterns in a viscous diffusion equation, Trans. Amer. Math. Soc., 324 (1991), pp. 331-351.

[19] M. Otsuji, S. Ishinara, C. Co, K. Kaibuchi, A. Mochizuki, and S. Kuroda, A mass conserved reaction-diffusion system captures properties of cell polarity, PLOS Computational Biology, 3 (2007), pp. 1-15.

[20] B. Perthame, Parabolic equations in biology, Lecture Notes on Mathematical Modelling in the Life Sciences, Springer, Cham, 2015.

[21] P. I. Plotnikov, Forward-backward parabolic equations and hysteresis, Zap. Nauchn. Sem. S.Peterburg. Otdel. Mat. Inst. Steklov. (POMI), 233 (1996), pp. 183-209, 257-258.

[22] J. Ranft, L. G. Almeida, P. C. Rodriguez, A. Triller, and V. Hakim, An aggregationremoval model for the formation and size determination of post-synaptic scaffold domains, PLOS Computational Biology, 13 (2017), pp. 1-18.

[23] T. O. Sakamoto, Hopf bifurcation in a reaction-diffusion system with conservation of mass, Nonlinearity, 26 (2013), p. 2027.

[24] K. Sekimoto And A. Triller, Compatibility between itinerant synaptic receptors and stable postsynaptic structure, Phys. Rev. E, 79 (2009), p. 031905. 
[25] N. Shigesada, K. Kawasaki, and E. Teramoto, Spatial segregation of interacting species, Journal of Theoretical Biology., 79 (1979), pp. 83-89.

[26] A. Terracina, Two-phase entropy solutions of forward-backward parabolic problems with unstable phase, Interfaces Free Bound., 17 (2015), pp. 289-315. 